\title{
Influencia de la masa muscular de miembros superiores en sujetos físicamente activos ${ }^{1}$
}

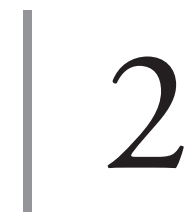

https://doi.org/10.21830/9789585284814.02

\author{
Brian Johan Bustos Viviescas', Andrés Alonso Acevedo Mindiola ${ }^{3}$, \\ Carlos Alberto Hernández Camargo ${ }^{4}$, Nelson Orlando Clavijo Gutiérrez', \\ Rafael Enrique Lozano Zapata ${ }^{6}$, José Alexander Ortiz Novoa 7 , \\ Leidy Estefanía Rodríguez Acuña ${ }^{8}$
}

\section{Resumen}

Objetivo: el propósito del presente estudio consistió en establecer la influencia de la masa muscular de miembros superiores durante la repetición máxima en press banca plano en sujetos físicamente activos. Metodología: estudio correlacional-exploratorio de enfoque cuantitativo y una muestra a conveniencia conformada por diez hombres (edad

1 El presente estudio es un resultado secundario de la investigación denominada "Desarrollo de la hipertrofia en los músculos de miembros inferiores a través de un programa de musculación”. El tipo de financiación es propia.

2 Docente de la Fundacion Universitaria Juan de Castellanos. Maestría en Actividad Física y Entrenamiento Deportivo - Universidad Montrer (en formación). Especialista en Métodos y Técnicas de Investigación - Fundación Universitaria Claretiana. Observatorio de Investigación en Ciencias de la Actividad Física y el Deporte. Contacto: bjbustos@jdc.edu.co. Orcid: https://orcid.org/0000-0002-4720-9018

3 Licenciado en Educación Básica con Énfasis en Educación Física, Recreación y Deportes de la Universidad de Pamplona (Sede Villa del Rosario). Contacto: andres.acevedo@unipamplona.edu.co. Orcid: https://orcid.org/0000-0003-0125-7265

4 Esp. en Entrenamiento Deportivo - Universidad de Pamplona. Director de Rendimiento Deportivo del Cúcuta Deportivo S.A. Contacto: carloshernandezc@unipamplona.edu.co. Orcid: https://orcid. org/0000-0001-7174-4806

5 Magíster en Ciencias de la Actividad Física, PhD. en Educación, Rector de Institución Educativa La Garita. Contacto: nelsonclavijo@usantotomas.edu.co. Orcid: https://orcid.org/0000-0002-1755-4830

6 Magíster en Educación Física mención en Fisiología del ejercicio. PhD. en Ciencias de la Actividad Física y el Deporte, docente de la Universidad de Pamplona (Sede Villa del Rosario). Contacto: rafaenloza@unipamplona.edu.co. Orcid: https://orcid.org/0000-0002-6239-5883

7 Licenciado en Educación Básica con Énfasis en Educación Física, Recreación y Deportes de la Universidad de Pamplona (Sede Villa del Rosario). Contacto: jose.novoa@unipamplona.edu.co. Orcid: https://orcid.org/0000-0003-1707-3719

8 Estudiante de la Licenciatura en Educación Básica con Énfasis en Educación Física, Recreación y Deportes de la Universidad de Pamplona. Contacto: leidy.rodriguez3@unipamplona.edu.co. Orcid: https:// orcid.org/0000-0001-7684-112X 
de $17,40 \pm 2,32$ años, talla de $172,10 \pm 5,76 \mathrm{~cm}$ y un peso corporal de $79,13 \pm 19,54 \mathrm{~kg}$ ) capacitados en el entrenamiento de la fuerza con sobrecargas. Se realizaron mediciones antropométricas para estimar la masa muscular de miembros superiores por medio de una ecuación y se evaluó la repetición máxima en el press banca plano empleando el protocolo propuesto por la National Strength and Conditioning Association. Por otra parte, para el análisis estadístico se utilizó el PSPP (licencia libre) para sistema operativo Windows 7, en el cual se evaluó la normalidad por medio de la prueba de Shapiro-Wilk y la correlación entre la masa muscular de miembros superiores con la repetición máxima en press banca, a través del coeficiente correlacional de Pearson. Resultados: después de analizar los datos obtenidos en esta investigación se puede identificar que la relación entre ambas variables es positiva pero no significativa $(r=0,35 ; \mathrm{p}=0,33)$. Conclusión: no existe relación significativa entre la masa muscular de miembros superiores y la repetición máxima en press banca plano, debido a la falta de experiencia deportiva en el trabajo con sobrecarga, al igual que la especificidad deportiva y la ganancia neta de masa muscular para generar un beneficio.

Palabras clave: entrenamiento de fuerza; fuerza muscular; prueba de ejercicio.

\section{Introducción}

Es evidente que el entrenamiento de la fuerza representa un factor crucial para el éxito competitivo (Galicia, 2014), pero a esto se debe sumar la necesidad de realizar valoraciones físicas para llevar a cabo un adecuado control y seguimiento del proceso de entrenamiento (Acevedo-Mindiola y Bustos-Viviescas, 2017), por ello, para alcanzar buenos resultados y optimizar el desempeño en competencias, es necesario evaluar la fuerza dentro del proceso de programación del entrenamiento deportivo.

Por otra parte, estudiar la asociación entre las características morfológicas en el deporte permitirá conocer la relación de la estructura física y el rendimiento deportivo (Lozano et al., 2017), no obstante, los estudios que buscan identificar cómo afectan las características morfológicas el rendimiento en los deportes de fuerza se caracterizan por evaluar la masa muscular total. Sin embargo, este parámetro no es lo suficientemente objetivo para establecer la distribución de la masa muscular con relación a las demandas de la práctica deportiva respecto a la masa muscular apendicular (Bustos-Viviescas et al., 2017), razón por la cual, se podría adoptar una serie de decisiones en la plani- 
ficación con relación a la optimización del rendimiento deportivo (González et al., 2015).

Teniendo en cuenta lo anterior, el press banca es uno de los principales ejercicios incluidos en el entrenamiento de la fuerza con sobrecargas en sujetos, entrenados o no entrenados, para optimizar su preparación y rehabilitación física, o para investigaciones científicas (Bustos-Viviescas et al., 2016). Además, se utiliza cuando el objetivo es medir la fuerza muscular de la parte superior del cuerpo (Kwon et al., 2011). De esta manera, es fundamental que este ejercicio sea incluido en todo programa de entrenamiento con sobrecargas que pretenda mejorar la fuerza y la hipertrofia musculares del miembro superior, en vista de que Figueiredo et al. (2016) indican que el orden de los ejercicios durante la sesión debe priorizar, al comienzo, aquellos ejercicios que más se requiere mejorar para permitir mayor volumen de entrenamiento y estimular una mayor ganancia de fuerza.

Sin embargo, la relación existente entre la masa muscular apendicular de miembros superiores y la repetición máxima en el press banca no están del todo claras, dado que, según Bustos-Viviescas et al. (2017), los estudios disponibles con respecto a esta temática se caracterizan por describir la masa muscular apendicular de diferentes disciplinas deportivas, pero no determinan la relación de este parámetro con la fuerza máxima y otros indicadores del rendimiento deportivo, por lo cual, se hace evidente que esta es una nueva línea de investigación que sirve para identificar cómo las características morfológicas afectan el rendimiento deportivo.

Ahora bien, entre las escasas investigaciones sobre el tema, se ha comprobado que la masa muscular de miembros inferiores no afecta el rendimiento del salto vertical —en la Selección Colombia de Gimnasia Artística (Hernández et al., 2018) — ni la capacidad de aceleración en línea recta —en árbitros de fútbol (Ortiz et al., 2018)—. Por otra parte, en miembros superiores se ha podido identificar que sí está relacionada con la fuerza prensil de mano (Bustos-Viviescas et al., 2018), pero no con la repetición máxima en press banca (Bustos-Viviescas et al., 2017). Por lo anterior, surge la necesidad de seguir realizando investigaciones de esta naturaleza con diferentes poblaciones, pues son pocos los estudios que aportan información valiosa sobre esta temática. 
Por todo lo expuesto anteriormente, se planteó la siguiente pregunta problema: ¿La masa muscular de miembros superiores influye en la repetición máxima en el press banca plano? Para tratar de dar respuesta a esta incógnita, el objetivo general del presente estudio se centró en establecer la influencia de la masa muscular de miembros superiores en la repetición máxima en press banca plano en sujetos físicamente activos, y para alcanzar el logro del mismo se plantearon los siguientes objetivos específicos: a) caracterizar a los participantes del estudio, b) aplicar las mediciones antropométricas y la repetición máxima en el press banca y c) relacionar la masa muscular de miembros superiores y la repetición máxima en el press banca.

\section{Metodología}

Diseño del estudio: estudio correlacional-exploratorio de enfoque cuantitativo y una muestra a conveniencia.

Participantes: sujetos capacitados en el entrenamiento de la fuerza con sobrecargas, puesto que asistían habitualmente a un centro de acondicionamiento físico o gimnasio.

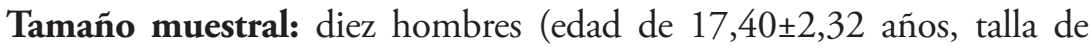
$172,10 \pm 5,76 \mathrm{~cm}$ y un peso corporal de 79,13 $\pm 19,54 \mathrm{~kg}$ ).

Métodos y procedimientos: entre los criterios de inclusión se encuentran la participación voluntaria y experiencia mínima de un año en el entrenamiento de la fuerza con sobrecargas; entre los criterios de exclusión se tuvo en cuenta el padecimiento de alguna lesión o enfermedad que afectara la fuerza de miembros superiores.

La masa muscular de miembros superiores (MMMs) fue estimada por medio de la ecuación desarrollada por Rodríguez, Almagià y Berral de la Rosa (2010) la cual se muestra a continuación:

$$
\text { MMMS }=(((T-P L T) \times(0,043 x(P B R) 2)) P) / 1000
$$

Donde, $\mathrm{T}=$ Talla PLT $=$ Pliegue tricipital $; \mathrm{PBR}=$ Perímetro brazo relajado; $\mathrm{P}=$ Peso corporal. 
La recolección de las mediciones antropométricas se realizó con los siguientes instrumentos:

- Báscula Tanita BC-730 (precisión de $100 \mathrm{~g}$ ).

- Tallímetro de pared Seca $206(0-220 \mathrm{~cm}$, precisión $1 \mathrm{~mm})$.

- Plicómetro Slim Guide (0-80 mm, precisión de 0,5 mm).

- Cinta antropométrica Seca $201(0-205 \mathrm{~cm}$, precisión $1 \mathrm{~mm})$.

Por otro lado, para evaluar la repetición máxima en el press banca plano se empleó el protocolo propuesto por la National Strength and Conditioning Association (NCAA) (Gregory \& Travis, 2015). El test finalizaba si se fallaba la repetición o si se ejecutaba sin la técnica correcta; el aumento de la carga fue de $5 \mathrm{~kg}$ de manera progresiva, para ello el descanso entre cada repetición fue lo suficientemente prolongado para garantizar la recuperación óptima del evaluado, así, el periodo de descanso fue de dos a tres minutos como se ha sugerido en otros estudios (Whisenant, Panton, East \& Broeder, 2003; Hutchins \& Gearhart, 2010).

Con anterioridad a la realización del test de $1 \mathrm{RM}$, se aplicó un calentamiento en el cual, en primer lugar, se efectuaba la movilidad articular y, posteriormente, se ejecutaba el press banca plano con pesos ligeros en 4 series de 15 repeticiones, con 2 minutos entre serie.

Plan de análisis: el análisis estadístico se realizó en PSPP (licencia libre) para sistema operativo Windows 7; con este software se evaluó la normalidad por medio de la prueba de Shapiro-Wilk y la correlación entre la masa muscular de miembros superiores con la repetición máxima en press banca, a través del coeficiente correlacional de Pearson.

Consideraciones éticas: para el desarrollo de esta investigación todos los participantes debían diligenciar un consentimiento informado por escrito, el cual tenía descrito las pruebas a ser realizadas, así como el alcance esperado de los resultados y los posibles riesgos durante el estudio. Igualmente, se consideraron los principios éticos para investigaciones en ciencias del deporte y el ejercicio (Harriss \& Atkinson, 2013); a este respecto, cabe señalar que este estudio cuenta con el aval del Comité de ética e impacto ambiental en inves- 
tigación de la Universidad de Pamplona, de acuerdo con el Acta N. ${ }^{\circ} 009$ del 28 de agosto de 2017.

\section{Resultados}

En la Tabla 1 se puede observar que las variables de estudio obtuvieron distribución normal de los datos por medio de la prueba aplicada $(\mathrm{p}>0,05)$.

Tabla 1. Prueba de normalidad

\begin{tabular}{lc}
\hline \multicolumn{1}{c}{ Variables } & Normalidad (Shapiro-Wilk) \\
\hline Masa muscular de miembros superiores $(\mathrm{kg})$ & 0,41 \\
Repetición máxima en press banca $(\mathrm{kg})$ & 0,12 \\
\hline
\end{tabular}

Fuente: elaboración propia.

En la Tabla 2 ambas variables se relacionaron de forma positiva, pero no significativamente $(r=0,35 ; \mathrm{p}>0,05)$. Del mismo modo, la Figura 1 muestra que existió una tendencia positiva entre la masa muscular de miembros superiores y la repetición máxima en press banca, lo que explica por qué aquellos sujetos que presentaron mayor masa muscular en este segmento tenían mejores resultados en el test de repetición máxima.

Tabla 2. Correlación entre variables

\begin{tabular}{lll}
\hline & \multicolumn{1}{c}{$\begin{array}{c}\text { Repetición máxima en } \\
\text { press banca }(\mathbf{k g})\end{array}$} \\
\hline $\begin{array}{l}\text { Masa muscular de } \\
\begin{array}{l}\text { miembros superiores } \\
(\mathrm{kg})\end{array}\end{array}$ & Coef. Pearson $(\mathrm{r})$ & 0,35 \\
\cline { 2 - 3 } & Sig. Bilateral $(\mathrm{p})$ & 0,33 \\
\hline
\end{tabular}

Fuente: elaboración propia. 


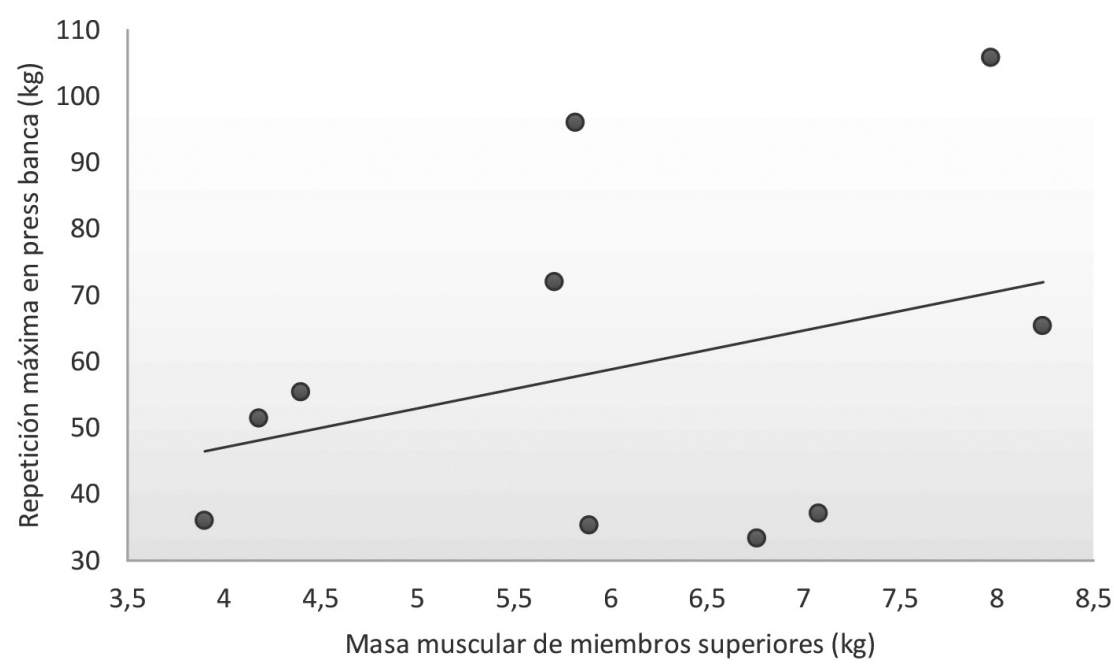

Figura 1. Dispersión con línea de tendencia.

Fuente: elaboración propia.

\section{Discusión}

El objetivo del presente estudio fue establecer la influencia de la masa muscular de miembros superiores en la repetición máxima en press banca plano en sujetos físicamente activos, en consecuencia, a partir de los resultados obtenidos en esta investigación, se puede identificar que la relación entre ambas variables es positiva pero no significativa $(r=0,35 ; \mathrm{p}=0,33)$.

Estudios similares que han evaluado la masa magra con la fuerza máxima en el tren superior por medio del press banca han identificado una relación positiva de 0,82 (Gómez et al., 2015a) y 0,44 (Gómez et al., 2015b), no obstante, solamente se conoce un estudio, realizado por Bustos-Viviescas et al. (2017), en el cual se relacionó la masa muscular de miembros superiores con la repetición máxima en el press banca y se obtuvo una asociación positiva en hombres físicamente activos con experiencia en el entrenamiento de la fuerza con sobrecargas $(r=0,52)$. Esto, a pesar de que las ganancias en la fuerza muscular están sustentadas en el aumento de la sección transversal del músculo esquelético y la coordinación intramuscular e intermuscular dada la importancia de la acti- 
vación y reclutamiento de unidades motoras en cada segmento implicado en el movimiento. Sin embargo, la literatura científica disponible hasta el momento respecto a esta temática es escasa, por lo que en sujetos físicamente activos que realizan entrenamiento de fuerza con sobrecargas la masa muscular de miembros superiores puede ser un factor que afecte de forma positiva el rendimiento de la fuerza máxima en el press banca.

Por otra parte, cabe resaltar que entre los factores principales para la adaptación al entrenamiento se encuentra la respuesta hormonal, ya que la testosterona y la hormona del crecimiento actúan de forma diferente dependiendo del estímulo mecánico y metabólico (Suárez, 2016). En consecuencia, los sujetos de este estudio asistían a un centro de acondicionamiento físico con el objetivo de lograr hipertrofia muscular, por tal razón desarrollaban grandes volúmenes de entrenamiento para generar una mayor lacticidemia en la musculatura (Bustos-Viviescas et al., 2017) y poder obtener una respuesta hipertrófica, debido a la mayor acidez (Buresh et al., 2009).

Así mismo, Gómez et al. (2015a) sugieren que desarrollar la fuerza es necesario para el logro de los objetivos de las diferentes disciplinas, especialmente en aquellas en la que esta capacidad es una característica predominante (halterofilia, powerlifting, entre otras), sin embargo, en los deportes cuyo fin es alcanzar elevados niveles de fuerza muscular, sin importar el tamaño muscular, existe una relación negativa entre la masa muscular apendicular y la repetición máxima, considerando que la tensión muscular alta produce adaptaciones neurales sin hipertrofia destacable (Vissing et al., 2008).

Cabe señalar en este punto que una de las principales limitaciones que debió afrontar la presente investigación consistió en la escasa muestra participante, a lo que debe sumarse que los participantes no realizaban actividad física de rendimiento para una disciplina deportiva específica. Igualmente, se debe considerar que no se pudo comparar la relación entre ambas variables en diferentes disciplinas deportivas. Por otra parte, resultaría interesante realizar en próximas investigaciones la evaluación de la masa muscular apendicular e incorporar a la evaluación de la fuerza muscular mediciones de electromiografía y velocidad del levantamiento, para posibilitar la asociación de la masa muscular a estas variables, determinantes del rendimiento en deportes cuyos 
niveles de fuerza no solo deben ser los más elevados, sino que también deben ser generados en el menor tiempo posible, como en el caso de los deportes de combate y velocidad.

\section{Conclusión}

No existe relación significativa entre la masa muscular de miembros superiores y la repetición máxima en press banca plano, esto, debido a la falta de experiencia deportiva en el trabajo con sobrecarga y la ganancia neta de la masa muscular tanto para generar un beneficio al activar las fibras de contracción rápida como para el buen desempeño durante la ejecución de estos movimientos, así como la falta de especificidad deportiva.

\section{Referencias}

Acevedo, A., \& Bustos, B. (2017). Correlación entre la flexibilidad de la musculatura isquiosural con la altura del salto vertical en jugadores de balonmano selección del departamento Norte de Santander. EDU-FÍSICA: Revista de Ciencias Aplicadas al Deporte, 9(20), 109-120. http://revistas.ut.edu.co/index.php/edufisica/article/view/1198/957

Buresh, R., Berg, K., \& French, J. (2009). The effect of resistive exercise rest interval on hormonal response, strength, and hypertrophy with training. Journal of Strength and Conditioning Association, 23(1), 62-71. https://doi.org/10.1519/JSC.0b013e318185f14a

Bustos, B., Lozano, R., \& Justacaro, G. (2016). Incremento de la fuerza dinámica máxima a través de un protocolo de acción recíproca con deportistas amateurs. Revista Impetus, 10(1-2), 119-126. http://revistaimpetus.unillanos.edu.co/impetus/index.php/Imp1/article/viewFile/165/134

Bustos, B., Acevedo, A., \& Rodríguez, L. (2017). Relación entre la masa muscular apendicular y la repetición máxima en sujetos físicamente activos. Kronos: Revista Científica de Actividad fisica y Deporte, 16(2). https://revistakronos.info/articulo/relacion-entre-la-masa-muscular-apendicular-y-la-repeticion-maxima-en-sujetos-fisicamente-activos-2366-sa-O5a57800957910/

Bustos, B., Rodríguez, L., Acevedo, A., Ortiz, J., Lozano, R., Durán, L., Bautista, V., \& Hernández, C. (2018). La masa muscular de miembros superiores está asociada a la fuerza prensil de la mano en sujetos físicamente activos. I Concurso de Investigación Acome, Universidad de Pamplona, Colombia.

Figueiredo, T., Miranda, H., Willardson, J., Schneider, A., Freitas de Salles, B., Spineti, J., Paz, G., Santana, H., \& Simão, R. (2016). Influencia del orden de los ejercicios en la determinación de cargas de una y diez repeticiones máximas. Revista Entrenamiento Deportivo, 
30(4). https://g-se.com/influencia-del-orden-de-los-ejercicios-en-la-determinacion-decargas-de-una-y-diez-repeticiones-maximas-2095-sa-d57cfb2727acf2

Galicia, A. (2014). Conceptos básicos sobre la fuerza muscular. Revista Digital EFDeportes, 18(190). http://www.efdeportes.com/efd190/conceptos-basicos-sobre-la-fuerza-muscular.htm

Gómez, J., García, J., Morales, I., Quintana, A., Quintana, L., \& Rivera, A. (2015a). Evaluación de la fuerza máxima y su relación con la masa muscular del tren superior de universitarios. Revista Digital EFDeportes, 20(207). http://www.efdeportes.com/efd207/fuerza-maximay-relacion-con-la-masa-muscular.htm

Gómez, J., Hernández, S., Marín, Z., \& Rivera, A. (2015b). Análisis de la composición corporal y manifestación de la fuerza máxima en estudiantes de educación física. Revista Digital EFDeportes, 20(207). http://www.efdeportes.com/efd207/composicion-corporal-y-fuerza-maxima.htm

González, J., Navarro, F., \& Pereira, P. (2015). La planificación del entrenamiento deportivo: cambios vinculados a las nuevas formas de entender las estructuras deportivas contemporáneas. Revista de Entrenamiento Deportivo, 29(1), 1-14. https://journal.onlineeducation. center/api-oas/v1/articles/sa-h57cfb2724acb7/export-pdf

Gregory, G., \& Travis, N. (2015). Essentials of Strength Training and Conditioning (4a. ed.). Human Kinetics.

Harriss, D., \& Atkinson, G. (2013). Ethical standards in sport and exercise science research: 2014 update. International Journal of Sports Medicine, 34(12), 1025-1028. http://dx.doi. org/10.1055/s-0033-1358756

Hernández, C., Bustos, B., Acevedo, A., Rodríguez, L., Ortiz, J., Lozano, R, Duran, L., \& Bautista, V. (2018). La masa muscular de miembros inferiores no afecta el rendimiento del salto vertical en la Selección Colombia de Gimnasia Artística. I Concurso de Investigación Acome, Universidad de Pamplona, Colombia.

Hutchins, M., \& Gearhart, R. (2010). Accuracy of 1-RM Prediction equations for the bench press and biceps curl. Journal of Exercise Physiology Online, 13(3), 32-39. https://www. researchgate.net/publication/289212637_Accuracy_of_1-RM_Prediction_equations_ for_the_bench_press_and_biceps_curl

Kwon, H., Han, K., Ahn, H., Lee, J., Park, G., \& Min, K. (2011). The correlations between extremity circumferences with total and regional amounts of skeletal muscle and muscle strength in obese women with type 2 diabetes. Diabetes \& Metabolism Journal, 35(4), 374-383. http://doi.org/10.4093/dmj.2011.35.4.374

Lozano, R., Bustos, B., Acevedo, A., \& Bautista, V. (2017). Composición corporal y somatotipo de los tenistas de mesa de Norte de Santander que participaron en los XX Juegos Nacionales, Colombia. EmásF: Revista Digital de Educación Física, 8(46), 50-60. http:// emasf.webcindario.com/Composicion_corporal_y_somatotitpo_de_los_tenistas_de_ mesa_de_Norte_de_Santander.pdf

Ortiz, J., Bustos, B., Rodríguez, L., Acevedo, A., Durán, L., Hernández, C., Bautista, V., \& Lozano, R. (2018). ¿La masa muscular de miembros inferiores es influyente en la capacidad de aceleración en línea recta en árbitros de fútbol? I Concurso de Investigación Acome, Universidad de Pamplona, Colombia. 
Rodríguez, F., Almagià, A., \& Berral de la Rosa, F. (2010). Estimación de la masa muscular de los miembros apendiculares, a partir de densitometría fotónica dual (DEXA). International Journal of Morphology, 28(4), 1205-1210. https://dx.doi.org/10.4067/ S0717-95022010000400034

Suárez, D. (2016). Testosterona y hormona del crecimiento: sistemas de entrenamiento de la fuerza. Revista de Entrenamiento Deportivo, 30(1), 1-11. https://journal.onlineeducation. center/api-oas/v1/articles/sa-a57cfb272780d3/export-pdf

Vissing, K., Brink, M., Lønbro, S., Sørensen, H., Overgaard, K., Danborg, K., Mortensen, J., Elstrøm, O., Rosenhøj, N., Ringgaard, S., Andersen, J., \& Aagaard, P. (2008). Muscle adaptations to plyometric vs. resistance training in untrained young men. Journal of Strength and Conditioning Research, 22(6), 1799-1810. https://doi.org/10.1519/ JSC.0b013e318185f673

Whisenant, M., Panton, L., East, W., \& Broeder, C. (2003). Validation of submaximal prediction equations for the one repetition maximum bench press test on a group of collegiate football players. Journal Strength and Conditioning Research, 17(2), 221-227. 